\title{
Technè
}

La science au service de l'histoire de l'art et de la préservation des biens culturels

47 | 2019

Bernard Palissy : nouveaux regards sur la céramique française auX $\mathrm{XVI}{ }^{\mathrm{e}}$ et $\mathrm{XVII}{ }^{\mathrm{e}}$ siècles

\section{Les céramiques post-palisséennes de provenance ancienne dans les collections françaises : analyse des glaçures}

Post-Palissian ceramics from previous collections in French public museums: an analysis of their glazes

\section{Anne Bouquillon}

\section{OpenEdition \\ Journals}

Édition électronique

URL : http://journals.openedition.org/techne/1558

DOI : 10.4000/techne.1558

ISSN : 2534-5168

\section{Éditeur}

C2RMF

\section{Édition imprimée}

Date de publication : 1 juin 2019

Pagination : 90-101

ISBN : 978-2-11-152830-7

ISSN : $1254-7867$

\section{Référence électronique}

Anne Bouquillon, « Les céramiques post-palisséennes de provenance ancienne dans les collections françaises : analyse des glaçures », Technè [En ligne], 47 | 2019, mis en ligne le 01 juin 2020, consulté le 27 juillet 2020. URL : http://journals.openedition.org/techne/1558; DOI : https://doi.org/10.4000/ techne.1558

\section{c.) (i) (9)}

La revue Technè. La science au service de l'histoire de l'art et de la préservation des biens culturels est mise à disposition selon les termes de la Licence Creative Commons Attribution - Pas d'Utilisation Commerciale - Pas de Modification 4.0 International. 


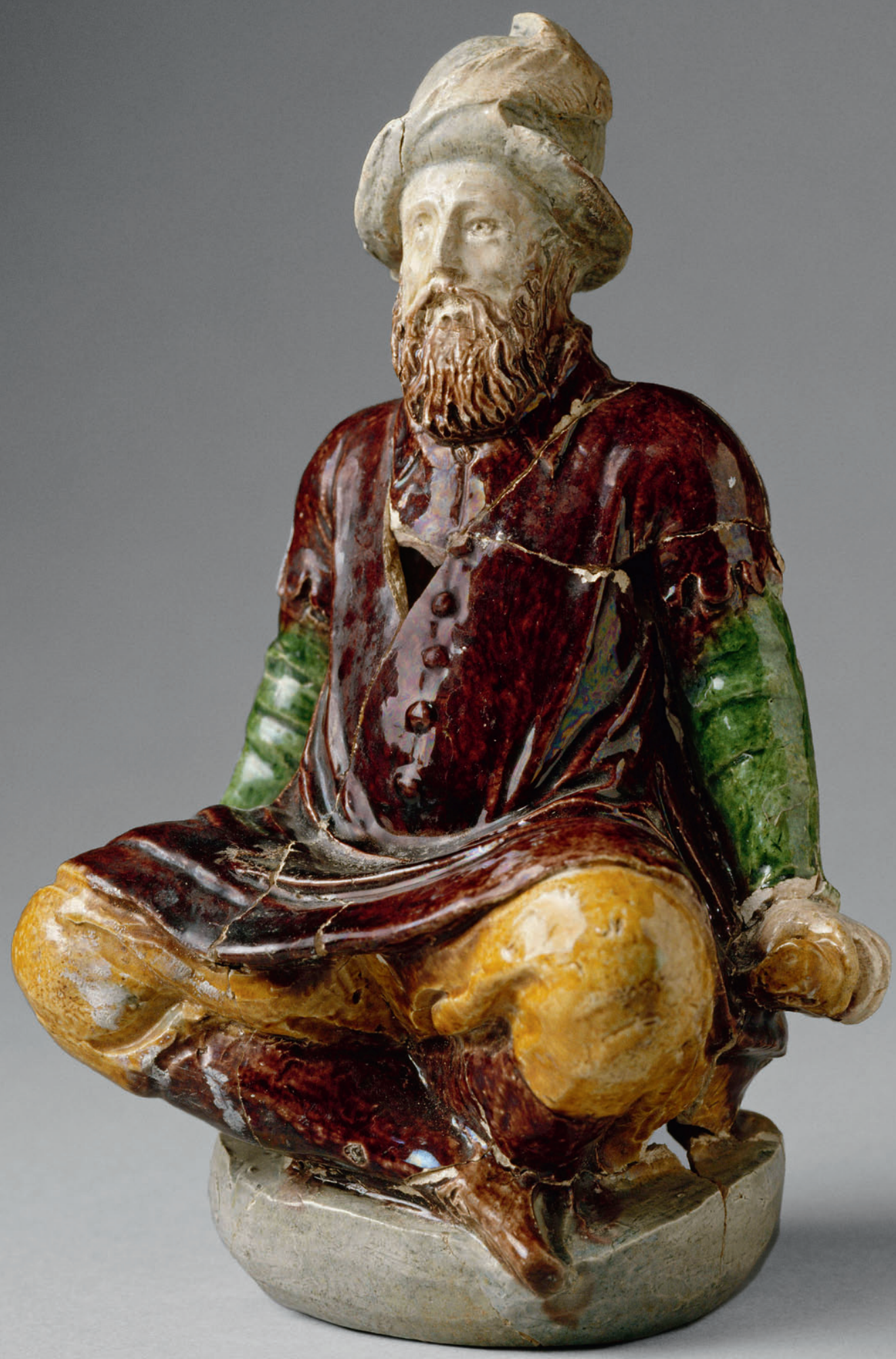

Fig. 1. Paris (?), première moitié du XviI ${ }^{\mathrm{e}}$ siècle, Statuette, Personnage masculin barbu assis en tailleur,

terre cuite à glaçure plombifère (H. 16,5 cm, L. 11,5 cm, 1. 7,7 cm), Paris, musée du Louvre, département des Objets d'art, OA 12072. @ RMN-Grand Palais (musée du Louvre)/Hervé Lewandowski. 
Anne Bouquillon

\section{Les céramiques post-palisséennes de provenance ancienne dans les collections françaises : analyse des glaçures}

Post-Palissian ceramics from previous collections in French public museums: an analysis of their glazes

Résumé. Les glaçures mises en cuvre par les céramistes postpalisséens sont dans l'ensemble assez homogènes. À partir de pièces dont la provenance est datée des XVII et XVIII ${ }^{e}$ siècles, nous en donnons les principales caractéristiques et tentons de dégager quelques différences afin de proposer des regroupements. Ce sont de faibles variations dans les compositions chimiques et la présence ou l'absence de certains éléments comme le bore qui conduisent à formuler des hypothèses sur la répartition des ateliers de production. Un bref aperçu est donné en outre sur les premiers résultats concernant un groupe de petits personnages, production post-palisséenne autrefois attribuée à Avon.

Mots-clés. Palissy, saisies révolutionnaires, glaçures plombifères, bore, PIXE, analyses chimiques, céramique post-palisséenne.
Abstract. The glazes used by post-Palissian potters are relatively homogeneous overall. Based on pieces dated with precision from the 17th and 18th centuries, we shall describe their principal characteristics and attempt to draw forth certain differences so as to propose groupings. The slight variations in chemical compositions, and the presence or absence of certain elements such as boron, are what have led us to formulate hypotheses on the separate production workshops. In addition, we shall give a brief overview of the first results concerning a group of small postPalissian figures, formerly thought to have been produced in Avon.

Keywords. Palissy, seized during the French Revolution, lead glazes, boron, PIXE, chemical analyses, post-Palissian ceramics.
Les recherches menées sur les productions sérielles de céramiques décoratives françaises, dites post-palisséennes ${ }^{1}$, ont été, jusqu'à un passé récent, peu nombreuses. Les premières conclusions que l'on pouvait en tirer apparaissaient imprécises, contestables, et cela pour plusieurs raisons. D'une part, la quasi-totalité de ces œuvres moulées présente des caractéristiques matérielles très voisines : pâte blanche, souvent fine, riche en aluminium et probablement kaolinitique, glaçures transparentes, peu épaisses, souvent tressaillées ${ }^{2}$, colorées par les oxydes traditionnels, cuivre pour le vert, cobalt pour le bleu, fer pour le jaune, manganèse pour le mauve et des mélanges de ces divers oxydes pour compléter la palette (brun, gris, nuances de vert, etc.). Ces couvertes sont toutes plombifères. Dans ces conditions de ressemblance, tout classement s'avère impossible malgré l'identification de deux paramètres semblant plus discriminants : la présence d'alcalins dans les glaçures bleues colorées au cobalt et les traces de bore (moins de $0,5 \% \mathrm{~B}_{2} \mathrm{O}_{3}$ en général, parfois jusqu'à $5 \%)^{3}$. D'autre part, en l'absence de tout objet dûment authentifié, il suffisait que les revers des pièces fussent marbrés ou que l'on y reconnût certaines caractéristiques des rustiques figulines (présence de coquilles, de végétaux, de petits animaux moulés) pour que l'on attribuât ces œuvres à l'atelier de Bernard Palissy, voire au maître lui-même. Ou bien, surtout à partir de la seconde moitié du XIX ${ }^{\mathrm{e}}$ et au $\mathrm{XX}^{\mathrm{e}}$ siècle, on les donnait à d'hypothétiques ateliers par exemple ceux de l'École de Fontainebleau, ceux d'Avon, ceux du Pré-d'Auge ou ceux de la Chapelle-des-Pots, sans que ces attributions reposent sur des bases scientifiques établies.

Mais depuis les travaux récents de Jessica Denis-Dupuis sur le cadre historique de ces productions, depuis les analyses physico-chimiques d'œuvres authentiques palisséennes trouvées en fouille et des céramiques postérieures, des différences sont décelables entre les œuvres de Palissy lui-même et celles plus tardives, et d'autres hypothèses peuvent être avancées ${ }^{4}$.

Dans cette masse importante d'œuvres, d'attributions, d'origines possibles, il est essentiel de pouvoir s'appuyer aussi sur des jalons chronologiques indiscutables. Or, jusqu'à présent, mis à part les productions d'Avisseau (1795-1861) ou de Pull (1810-1889) signées par les artistes, il n'existait que très 
Tableau 1. Liste des œuvres étudiées

\begin{tabular}{|c|c|c|c|}
\hline Musée & $\mathrm{N}^{\circ}$ d'inventaire & Cuvre & Collection \\
\hline \multicolumn{4}{|l|}{ 1a. Ensemble REF } \\
\hline $\begin{array}{l}\text { Musée du Louvre - } \\
\text { Département des Objets d'art }\end{array}$ & MR 3528 & $\begin{array}{l}\text { Bassin d'aiguière } \\
\text { dit « de la Tempérance » }\end{array}$ & $\begin{array}{l}\text { Saisie napoléonienne } \\
\text { Coll. Brunswick }\end{array}$ \\
\hline \multirow[t]{2}{*}{ Musée des Beaux-Arts de Rennes } & 794.1 .570 & Aiguière à décor rustique & $\begin{array}{l}\text { Saisie révolutionnaire } \\
\text { Coll. De Robien }\end{array}$ \\
\hline & 794.1 .569 & Plat ovale à décor rustique & $\begin{array}{l}\text { Saisie révolutionnaire } \\
\text { Coll. De Robien }\end{array}$ \\
\hline \multirow[t]{4}{*}{ Musée de Picardie - Amiens } & MP 1876.290 & $\begin{array}{l}\text { Plat ovale orné de deux anges } \\
\text { et d'une couronne }\end{array}$ & $\begin{array}{l}\text { Saisie révolutionnaire } \\
\text { Abbaye Saint-Jean-des- } \\
\text { Prémontrés }\end{array}$ \\
\hline & MP 1876.304 bis & $\begin{array}{l}\text { Rocher rustique à têtes } \\
\text { de murènes, lézards et } \\
\text { coquillages, surmonté } \\
\text { d'une statuette représentant } \\
\text { un Joueur de cornemuse }\end{array}$ & $\begin{array}{l}\text { Saisie révolutionnaire } \\
\text { Abbaye Saint-Jean-des- } \\
\text { Prémontrés }\end{array}$ \\
\hline & MP 273 & Neptune sur un cheval marin & $\begin{array}{l}\text { Saisie révolutionnaire } \\
\text { Abbaye Saint-Jean-des- } \\
\text { Prémontrés }\end{array}$ \\
\hline & MP 1876.295 & $\begin{array}{l}\text { Grand plat rond avec Persée et } \\
\text { Andromède et huit cavités sur } \\
\text { l'aile }\end{array}$ & $\begin{array}{l}\text { Saisie révolutionnaire } \\
\text { Abbaye Saint-Jean-des- } \\
\text { Prémontrés }\end{array}$ \\
\hline \multicolumn{4}{|l|}{ 1b. Statuettes } \\
\hline \multirow{4}{*}{$\begin{array}{l}\text { Musée du Louvre } \\
\text { - Département des Objets d'art }\end{array}$} & OA 12071 & Statuette de chien sur socle & Fouilles \\
\hline & OA 12072 & $\begin{array}{l}\text { Personnage masculin barbu } \\
\text { assis en tailleur }\end{array}$ & Fouilles \\
\hline & MR 2368 & Statuette de vache & Coll. Durand \\
\hline & MR 2370 & Statuette de Capitan & Coll. Durand \\
\hline $\begin{array}{l}\text { Musée national de la Renaissance - } \\
\text { Écouen }\end{array}$ & E.Cl. 13199 & Joueur de cornemuse & Coll. Durand \\
\hline
\end{tabular}

peu de références datées. L'iconographie servait de repère : ainsi, des représentations d'Henri IV et de sa famille, ou du couronnement de Louis XIII permettaient de proposer des datations post-quem toutes relatives. C'est pourquoi la mention d'œuvres précises dans les registres de saisies révolutionnaires napoléoniennes, œuvres identifiées dans les collections de musées français, constitue désormais des jalons chronologiques essentiels pour asseoir ou moduler nos hypothèses de travail ${ }^{5}$. Après leur étude en laboratoire, nous pourrons donc confronter les données obtenues aux propositions formulées dans deux articles précédents ${ }^{6}$. Nous les enrichirons par l'analyse d'une série de petits sujets plastiques (fig. 1) conservés aux musées d'Écouen et du Louvre dont l'origine reste incertaine mais qui étaient habituellement aussi associés aux productions dites « d'Avon » ou de Fontainebleau.
Ces études pluridisciplinaires nouvelles couplant l'histoire des collections, l'étude historique des œuvres, l'identification des fossiles présents dans les décors et l'analyse des matériaux constitutifs permettent de proposer des critères de différenciation plus précis et de proposer des éléments solides sur l'origine si controversée de ces productions sérielles.

\section{Matériel étudié et protocoles analytiques}

À un corpus précédemment étudié de 27 pièces, que nous appellerons par la suite Corpus $\mathrm{I}^{7}$, vont s'ajouter deux nouveaux ensembles dont les glaçures ont fait l'objet d'analyses chimiques (tableau 1) : d'abord sept œuvres de référence provenant des saisies révolutionnaires et napoléoniennes, puis 
cinq petites statuettes pour augmenter le corpus de comparaison. Le premier de ces nouveaux ensembles (ensemble REF, tableau 1 a) est composé de deux pièces conservées au musée des BeauxArts de Rennes, un plat ovale (794.1.569) (voir fig. 20 a et b du Corpus des céramiques post-palisséennes dans ce volume) et une aiguière à décor rustique (794.1.570) (voir fig. $21 \mathrm{du}$ Corpus des céramiques post-palisséennes dans ce volume) ; s'y ajoutent quatre œuvres du musée des Beaux-Arts d'Amiens : l'étonnant rocher rustique, ou fontaine, surmonté d'une statuette de joueur de cornemuse (MP 1876.304 bis) (voir fig. 2 de l'article de F. Barbe $e t$ al. dans ce volume), un plat ovale orné de deux anges (MP 1876.290) (voir fig. 15 a et b du Corpus des céramiques post-palisséennes dans ce volume), le grand plat illustrant Persée et Andromède (MP 1876.295) (voir fig. $14 \mathrm{a}$ et b du Corpus des céramiques post-palisséennes dans ce volume) et la statuette de Neptune sur un cheval marin (MP 273) (fig. 2), ainsi qu'un bassin d'aiguière dit « de la Tempérance » (MR 3528) de la collection Brunswick conservé au Louvre (voir fig. 9 a et b du Corpus des céramiques post-palisséennes dans ce volume).

Le second ensemble (tableau 1 b) regroupe quant à lui cinq statuettes : deux pièces retrouvées lors des fouilles du Grand Louvre, soit une statuette de petit chien sur socle (OA 12071) (fig. 3) et un personnage masculin barbu assis en tailleur (OA 12072) (fig. 1), ainsi que trois autres œuvres de la collection Durand entrée au Louvre en 1825, sans provenance antérieure, une vache (MR 2368) (fig. 4), un joueur de cornemuse (E.Cl. 13199) (voir fig. 1 de l'article de P. Lehuédé et al. dans ce volume) et une statuette de Capitan (MR 2370) (fig. 5).

Les pièces étant le plus souvent intactes, seules des méthodes analytiques entièrement non destructives étaient envisageables. C'est pourquoi les analyses par faisceau d'ions, PIXE (Particle-Induced X-ray Emission) et PIGE (ParticleInduced Gamma-ray Emission) ont été choisies. Nous ne pourrons pas présenter dans cet article l'analyse des pâtes, encore trop parcellaire compte tenu du trop petit nombre de prélèvements possibles. Seules les glaçures seront étudiées.

Le protocole utilisé est celui mis en œuvre lors de nos études précédentes, afin que les résultats soient comparables $^{8}$.

Une remarque préalable s'impose. La forme des œuvres étudiées, parfois trop creuse ou trop grande, a pu constituer un frein pour l'analyse de certaines plages colorées. En effet, la structure du système de détection et de sortie du faisceau d'ions d'AGLAE interdit l'approche à moins de $1 \mathrm{~mm}$, indispensable à la détection des éléments chimiques. C'est pourquoi, sur certains plats, toutes les couleurs n'ont pu être analysées.

Des analyses par MEB-EDX de coupes stratigraphiques allant de la glaçure à la pâte, sur des œuvres comparables de collections françaises ou américaines, ont montré que l'épaisseur des glaçures est en général supérieure à $150 \mu \mathrm{m}^{9}$. Les informations obtenues par des faisceaux de protons de $3 \mathrm{MeV}$ proviennent d'une profondeur maximale de $50 \mu \mathrm{m}$ dans le matériau : avec les conditions analytiques choisies, les données correspondent donc uniquement à la glaçure et ne sont perturbées ni par la pâte sous-jacente ni même par l'interface pâte/glaçure.

\section{Résultats sur les œuvres de référence}

Les sept œuvres (ensemble REF), mentionnées dans les registres de saisies révolutionnaires ou napoléoniennes, sont évidemment antérieures au XIX ${ }^{\mathrm{e}}$ siècle. Toutes les glaçures de cet ensemble REF contiennent du bore, en plus ou moins faibles teneurs. La présence de cet élément n'est pas compatible, dans l'état actuel des connaissances, avec une production Renaissance, donc palisséenne ${ }^{10}$. Ces œuvres appartiennent donc à ce qu'il est convenu d'appeler les céramiques post-palisséennes. Sans surprise, leur analyse révèle les compositions classiques mentionnées dès le début de cet article. Il s'agit pour l'ensemble des couleurs, sauf pour le bleu, de verres très plombifères, transparents (entre 50 et 
Tableau 2. Composition chimique des glaçures transparentes

- Données PIXE exprimées en \% d'oxydes - ND = Non détecté

\begin{tabular}{|l|l|l|l|l|l|l|l|l|l|l|l|l|}
\hline & $\mathrm{N}^{\circ}$ d'inventaire & $\mathrm{Na}_{2} \mathrm{O}$ & $\mathrm{MgO}^{\prime}$ & $\mathrm{Al}_{2} \mathrm{O}_{3}$ & $\mathrm{SiO}_{2}$ & $\mathrm{~K}_{2} \mathrm{O}$ & $\mathrm{CaO}$ & $\mathrm{TiO}_{2}$ & $\mathrm{Fe}_{2} \mathrm{O}_{3}$ & $\mathrm{SnO}_{2}$ & $\mathrm{PbO}$ & $\mathrm{B}_{2} \mathrm{O}_{3}$ \\
\hline Gp 1 & MP 1876.290 & 1,2 & 0,6 & 2,6 & 38,6 & 1,2 & 3,1 & 0,5 & 1,0 & 0,0 & 50,3 & \\
\hline & MP 1876.304 bis & 0,2 & 0,3 & 3,6 & 42,5 & 0,8 & 2,5 & 0,4 & 0,9 & $\mathrm{ND}$ & 47,9 & 0,2 \\
\hline & MR 3528 & 1,1 & 0,3 & 2,2 & 44,1 & 0,7 & 1,4 & 0,2 & 0,4 & $\mathrm{ND}$ & 48,5 & 2,3 \\
\hline & 794.1 .569 & 0,5 & 0,5 & 3,1 & 43,9 & 0,7 & 2,2 & 0,3 & 0,7 & $\mathrm{ND}$ & 47,3 & 1,5 \\
\hline Aiguière & 794.1 .570 & 1,0 & 0,6 & 2,8 & 41,5 & 1,2 & 1,0 & 0,3 & 0,3 & $\mathrm{ND}$ & 49,6 & 6 \\
\hline Gp 2 & MP 273 & 0,2 & 0,2 & 1,1 & 29,1 & 0,3 & 1,1 & 0,2 & 0,2 & $\mathrm{ND}$ & 66,5 & $<0,1$ \\
\hline & $\mathrm{MP} \mathrm{1876.295}$ & 0,2 & 0,1 & 1,7 & 26,3 & 0,3 & 0,4 & 0,2 & 1,0 & 0,4 & 67,9 & $<0,1$ \\
\hline
\end{tabular}

$70 \% \mathrm{PbO})$, siliceux $\left(30-50 \% \mathrm{SiO}_{2}\right)$ et plus ou moins alumineux. Tous contiennent de faibles teneurs en bore, souvent inférieures à $0,5 \% \mathrm{~B}_{2} \mathrm{O}_{3}$, sauf pour l'aiguière du musée de Rennes (plus de $10 \%$ dans la glaçure bleue) ; mais cet élément n'est pas réparti de manière homogène d'une couleur à l'autre ${ }^{11}$. Sodium, potassium, calcium et magnésium sont présents en très faibles quantités. Les éléments colorants sont toujours les mêmes, fer, cuivre, manganèse, cobalt, respectivement pour le jaune, le vert, le brun ou le violet, et pour le bleu. Des proportions variables de ces oxydes en mélange permettent d'obtenir une palette de couleurs plus étendue : gris, différentes nuances de bruns, de verts, de bleus. Le blanc correspond en réalité à une glaçure transparente posée sur une pâte très blanche. Nous n'avons trouvé aucune glaçure opacifiée à l'étain dans les différents objets étudiés.
Ces observations générales étant faites, nous nous proposons de reprendre chaque couleur séparément afin de mettre en évidence d'éventuelles différences au sein de l'ensemble REF et de comparer les résultats avec ceux déjà recueillis pour le Corpus I et publiés dans les deux articles d'Archaeometry.

\section{Les glaçres transparentes incolores}

Le rapport $\mathrm{PbO} / \mathrm{SiO}_{2}$ permet de distinguer deux groupes (tableau 2) :

- le premier (quatre œuvres) est caractérisé par un rapport voisin de 1,1 ; les teneurs en alumine, en alcalins et en magnésium sont légèrement plus élevées que dans le groupe suivant.

- le second ne contient que deux pièces (le Neptune MP 273 et un grand plat MP 1876.295) : le rapport $\mathrm{PbO} / \mathrm{SiO}_{2}$ est supérieur à 2 , la teneur en plomb dépasse $65 \% \mathrm{PbO}$ en moyenne. On notera dans le plat la présence de très faibles teneurs en étain, bien insuffisantes toutefois pour créer une quelconque opacification.

Une des œuvres se distingue nettement des six précédentes, l'aiguière de Rennes (794.1.570), dont la glaçure contient près de $7 \% \mathrm{~B}_{2} \mathrm{O}_{3}$.

En comparant ces données avec celles déjà publiées, on constate que les œuvres du premier groupe sont proches de celles du Corpus I.

Fig. 3. Paris (?), première moitié du XviI siècle, Statuette, Chien sur un socle, terre cuite à glaçure plombifère (H. $3 \mathrm{~cm}, \mathrm{~L} .5,5 \mathrm{~cm}, 1.2,2 \mathrm{~cm})$, Paris, musée du Louvre, département des Objets d'art, OA 12071. (C) C2RMF/A. Maigret. 


\section{Les glaçures jaunes}

Toutes ces glaçures sont très riches en plomb (de 50 à $70 \% \mathrm{PbO})$. Elles sont toutes colorées par des teneurs notables en oxydes de fer (entre 3 et $8 \% \mathrm{Fe}_{2} \mathrm{O}_{3}$ ).

Il est intéressant de constater que deux « recettes », ou du moins deux compositions différentes coexistent dans l'ensemble REF (tableau 3).

Dans la première recette, qui concerne trois pièces, le fer est associé à de fortes teneurs en aluminium ( 5 à $7 \% \mathrm{Al}_{2} \mathrm{O}_{3}$ ) et, dans une moindre mesure, à du titane $\left(0,5 \% \mathrm{TiO}_{2}\right.$ en moyenne), alors que pour les trois autres pièces, dont MP 273 et MP 1876.295, l'aluminium ne dépasse pas $3 \%$. Aucune glaçure ne contient suffisamment d'antimoine pour que l'on puisse soupçonner la présence d'antimoniates de plomb, colorant très fréquent des glaçures jaunes des faïences à la Renaissance. On soulignera le fort pourcentage de fer (plus de $7 \% \mathrm{Fe}_{2} \mathrm{O}_{3}$ en moyenne) dans le jaune du Neptune (MP 273).

Les pièces du Corpus I présentent, pour cette couleur, des gammes de concentrations très habituelles proches de celles décrites ci-dessus : fortes teneurs en plomb, en aluminium et 3 à $5 \%$ d'oxyde de fer. Plus anecdotiques, quelques plages jaunes contiennent des teneurs en arsenic non négligeables sans qu'une explication crédible puisse pour le moment être avancée ; ce phénomène avait déjà été souligné pour d'autres pièces ${ }^{12}$.

\section{Les glaçures vertes}

Elles sont toutes colorées par l'ajout de cuivre auquel est associé du zinc. Les compositions globales sont assez homogènes, mis à part la présence dans quelques œuvres de traces d'étain ou d'antimoine et, pour le vert de l'aiguière de Rennes, des teneurs en bore supérieures aux autres. Aucun élément ne permet de parler d'une réelle palette de verts (tableau 4).

En revanche, on pourra mettre en évidence de nettes différences dans le rapport $\mathrm{Cu} / \mathrm{Zn}$ qui évolue entre 2 et 35 . En l'état de nos connaissances, ces disparités peuvent être liées à l'utilisation de métaux différents pour obtenir le cuivre nécessaire à la coloration de la glaçure : cuivre, laiton, bronze, etc.

Les glaçures vertes des pièces du Corpus I présentent les mêmes caractéristiques. On notera en outre, dans les trois représentations de Henri IV et sa famille (OA 1351, MNC 6016, m. 86-14) un colorant vert ne contenant que du cuivre sans autres éléments liés.

\section{Les glaçres violettes et brunes}

Bruns et violets sont surtout obtenus par le manganèse, parfois en concentrations très élevées (plus de $5 \% \mathrm{MnO}$ ). Les teneurs les plus importantes en $\mathrm{MnO}$ sont détectées pour le Neptune (MP 273). À l'exception du plat de Rennes (794.1.569), où le manganèse semble associé au baryum dans le brun mais pas dans le violet, aucune relation directe avec un autre élément n'est enregistrée. On notera des proportions variables en cobalt, cuivre, fer qui sont autant d'ajouts permettant de varier les nuances de brun ou de violet (tableau 5).

Nous avions pu faire les mêmes observations sur les pièces du Corpus I.

\section{Les glaçures bleues}

C'est dans les bleus que les compositions sont les plus variables (tableau 6). En effet, contrairement aux autres couleurs, avant la fin du XvirI ${ }^{\mathrm{e}}$ siècle, époque à laquelle le cobalt est connu en tant qu'élément chimique, l'oxyde colorant métallique n'est pas ajouté directement au mélange glaçurant, mais sous forme d'une fritte de verre alcaline ou calco-alcaline. Les éléments $\mathrm{Na}, \mathrm{Mg}$, K et Ca s'y rencontrent donc en concentrations plus importantes que dans l'ensemble des autres couleurs.

Quelles que soient les teneurs en alcalins et alcalinoterreux, le rapport $\mathrm{Na}+\mathrm{K} / \mathrm{Mg}+\mathrm{Ca}$ reste globalement le même (proche de 1), ce qui indiquerait que la matière première utilisée est la même, mais avec des effets de dilution différents, correspondant à l'intensité de la couleur recherchée. Deux

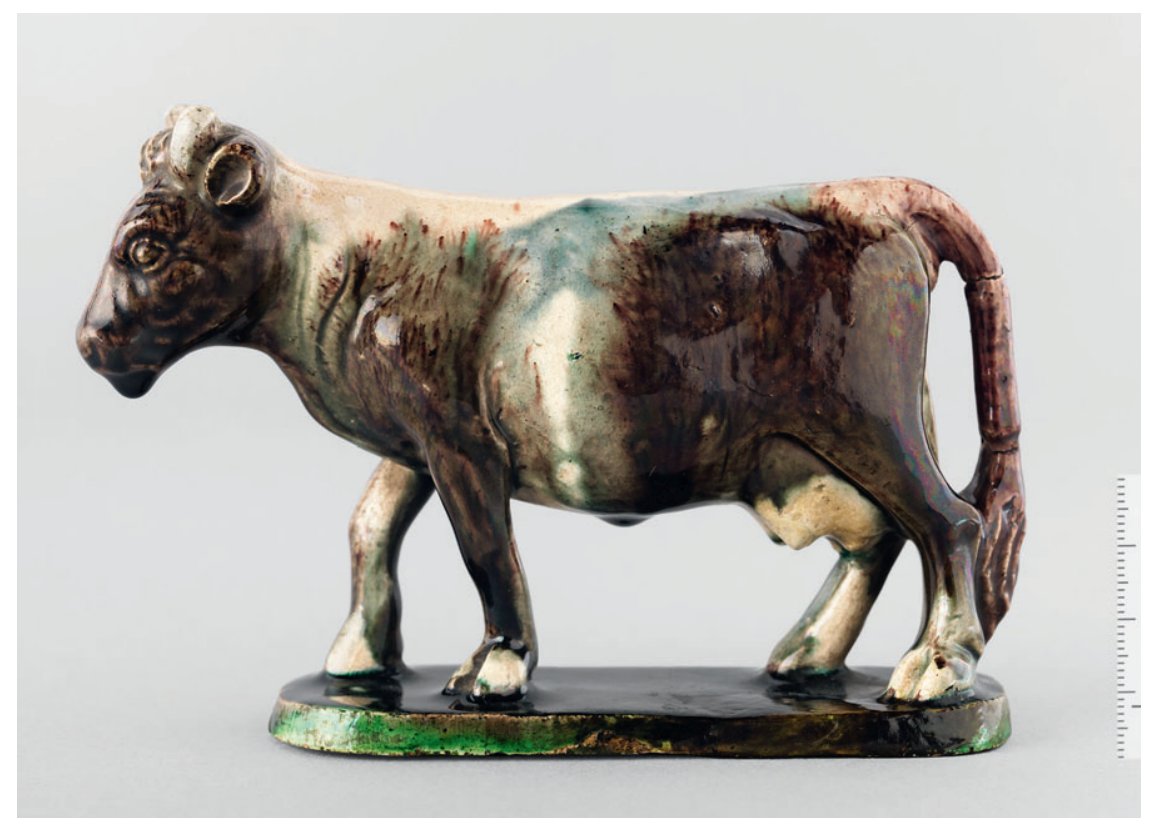

Fig. 4. Paris (?), première moitié du XvII ${ }^{\mathrm{e}}$ siècle, Statuette, Vache, terre cuite à glaçure plombifère $($ H. $6 \mathrm{~cm}, \mathrm{~L} .12,5 \mathrm{~cm}, 1.4 \mathrm{~cm})$, Paris, musée du Louvre, département des Objets d'art, MR 2368. (C) C2RMF/A. Maigret. 
Tableau 3. Composition chimique des glaçures jaunes

- Données PIXE exprimées en \% d'oxydes

\begin{tabular}{|c|c|c|c|c|c|c|c|c|c|c|c|c|c|c|}
\hline $\mathrm{N}^{\circ} \mathrm{d}^{\prime}$ inventaire & $\mathrm{Na}_{2} \mathrm{O}$ & $\mathrm{MgO}$ & $\mathrm{Al}_{2} \mathrm{O}_{3}$ & $\mathrm{SiO}_{2}$ & $\mathrm{~K}_{2} \mathrm{O}$ & $\mathrm{CaO}$ & $\mathrm{TiO}_{2}$ & $\mathrm{MnO}$ & $\mathrm{Fe}_{2} \mathrm{O}_{3}$ & $\mathrm{As}_{2} \mathrm{O}_{5}$ & $\mathrm{SnO}_{2}$ & $\mathrm{Sb}_{2} \mathrm{O}_{5}$ & $\mathrm{PbO}$ & $\mathrm{B}_{2} \mathrm{O}_{3}$ \\
\hline MP 1876.290 & 0,4 & 0,3 & 4,9 & 25,4 & 0,5 & 1,5 & 0,5 & 0,1 & 4,6 & 0,5 & 0,0 & 0,1 & 60,5 & \\
\hline 794.1 .569 & 0,4 & 0,3 & 6,9 & 35,1 & 1,0 & 1,5 & 0,5 & 0,1 & 4,0 & 0,0 & ND & 0,1 & 49,3 & 0,6 \\
\hline MP 1876.304 bis & 0,2 & 0,2 & 6,2 & 24,8 & 1,4 & 1,6 & 0,4 & 0,1 & 3,8 & 0,0 & 0,2 & 0,0 & 60,3 & 0,15 \\
\hline MR 3528 & 0,4 & 0,3 & 3,2 & 32,7 & 0,7 & 1,1 & 0,3 & 0,0 & 3,9 & 0,1 & ND & 0,2 & 56,0 & $0,5-1,6$ \\
\hline MP 1876.295 & 0,5 & 0,3 & 2,9 & 19,0 & 0,6 & 1,5 & 0,2 & 0,1 & 3,8 & 0,1 & 0,8 & 0,0 & 69,3 & 0,4 \\
\hline MP 273 & 0,5 & 0,5 & 1,9 & 21,8 & 0,8 & 3,9 & 0,2 & 0,5 & 7,1 & 0,2 & 0,0 & 0,2 & 61,4 & $<0,1$ \\
\hline
\end{tabular}

Tableau 4. Composition chimique des glaçures vertes - Données PIXE exprimées en \% d'oxydes

* Aiguière de Rennes comportant du bore

\begin{tabular}{|l|l|l|l|l|l|l|l|l|l|l|l|l|l|l|l|l|l|l|}
\hline $\mathrm{N}^{\circ}$ d'inventaire & $\mathrm{Na}_{2} \mathrm{O}$ & $\mathrm{MgO}$ & $\mathrm{Al}_{2} \mathrm{O}_{3}$ & $\mathrm{SiO}_{2}$ & $\mathrm{P}_{2} \mathrm{O}_{5}$ & $\mathrm{Cl}$ & $\mathrm{K}_{2} \mathrm{O}$ & $\mathrm{CaO}$ & $\mathrm{TiO}_{2}$ & $\mathrm{MnO}^{\prime}$ & $\mathrm{Fe}_{2} \mathrm{O}_{3}$ & $\mathrm{CuO}$ & $\mathrm{ZnO}$ & $\mathrm{As}_{2} \mathrm{O}_{5}$ & $\mathrm{SnO}_{2}$ & $\mathrm{Sb}_{2} \mathrm{O}_{5}$ & $\mathrm{PbO}^{\prime}$ & $\mathrm{B}_{2} \mathrm{O}_{3}$ \\
\hline MP 1876.290 & 0,00 & 0,52 & 5,01 & 25,11 & 0,09 & 0,42 & 0,47 & 2,08 & 0,67 & 0,11 & 1,02 & 8,21 & 0,72 & 0,31 & 0,19 & 0,06 & 54,89 & \\
\hline MP 1876-304 bis & 0,06 & 0,40 & 3,91 & 23,56 & 0,41 & 0,69 & 0,85 & 1,92 & 0,34 & 0,19 & 0,71 & 6,97 & 0,36 & 0,07 & 0,31 & 0,10 & 59,03 & 0,15 \\
\hline MP 273 & 0,26 & 0,43 & 1,28 & 28,24 & 0,15 & 1,07 & 1,37 & 1,81 & 0,17 & 0,20 & 1,41 & 2,82 & 0,54 & 0,57 & 0,28 & 0,20 & 58,69 & 0,2 \\
\hline MP 1876.295 & 0,24 & 0,50 & 3,50 & 25,15 & 0,12 & 0,68 & 0,73 & 1,39 & 0,26 & 0,07 & 0,51 & 3,96 & 0,21 & 0,08 & 0,89 & 0,19 & 61,44 & 0,3 \\
\hline MR 3528 & 0,31 & 0,34 & 3,20 & 33,22 & 0,08 & 1,37 & 0,79 & 1,41 & 0,24 & 0,09 & 1,21 & 2,14 & 0,30 & 0,29 & $\mathrm{ND}$ & 0,47 & 54,36 & 0,9 \\
\hline 794.1.569 & 0,04 & 0,31 & 4,98 & 26,90 & 0,05 & 0,25 & 0,55 & 1,09 & 0,39 & 0,06 & 0,57 & 5,53 & 0,32 & 0,18 & $\mathrm{ND}$ & 0,10 & 58,51 & 0,5 \\
\hline 794.1.570* & 0,50 & 0,56 & 3,99 & 17,79 & 0,35 & 0,54 & 1,21 & 3,24 & 0,40 & 0,03 & 0,46 & 4,12 & 1,56 & 0,00 & $\mathrm{ND}$ & 0,13 & 63,62 & $2-5$ \\
\hline
\end{tabular}

Tableau 5. Composition chimique des glaçures violettes et brunes

- Données PIXE exprimées en \% d'oxydes

\begin{tabular}{|l|l|l|l|l|l|l|l|l|l|l|l|l|l|l|l|l|l|}
\hline $\mathrm{N}^{\circ}$ d'inventaire & $\mathrm{Na}_{2} \mathrm{O}$ & $\mathrm{MgO}$ & $\mathrm{Al}_{2} \mathrm{O}_{3}$ & $\mathrm{Si}_{2}$ & $\mathrm{Cl}$ & $\mathrm{K}_{2} \mathrm{O}$ & $\mathrm{CaO}$ & $\mathrm{TiO}_{2}$ & $\mathrm{MnO}^{\prime}$ & $\mathrm{Fe}_{2} \mathrm{O}_{3}$ & $\mathrm{CoO}$ & $\mathrm{NiO}$ & $\mathrm{CuO}_{2}$ & $\mathrm{As}_{2} \mathrm{O}_{5}$ & $\mathrm{SnO}_{2}$ & $\mathrm{PbO}_{2}$ & $\mathrm{~B}_{2} \mathrm{O}_{3}$ \\
\hline MP 1876.290 & 0,94 & 0,54 & 3,37 & 34,75 & 0,37 & 1,15 & 2,57 & 0,46 & 2,19 & 1,39 & 0,61 & 0,39 & 0,12 & 0,63 & 0,00 & 50,31 & \\
\hline MP 273 & 0,52 & 0,39 & 1,79 & 25,96 & 0,99 & 0,75 & 2,73 & 0,19 & 4,65 & 0,62 & 0,05 & 0,11 & 0,14 & 0,09 & 0,19 & 60,22 & 0,1 \\
\hline MP 1876.295 & 0,30 & 0,30 & 1,07 & 25,77 & 0,90 & 0,35 & 1,03 & 0,11 & 1,89 & 1,23 & 0,17 & 0,14 & 0,20 & 0,25 & 2,15 & 63,89 & 0,1 \\
\hline MR 3528 & 0,91 & 0,45 & 2,68 & 37,09 & 1,36 & 0,87 & 1,64 & 0,18 & 1,78 & 0,44 & 0,08 & 0,07 & 0,35 & 0,16 & $\mathrm{ND}$ & 51,60 & $0,7-2,5$ \\
\hline 794.1.569 & 0,58 & 0,23 & 3,13 & 29,93 & 0,85 & 0,74 & 0,98 & 0,25 & 2,63 & 0,42 & 0,04 & 0,10 & 0,17 & 0,16 & $\mathrm{ND}$ & 59,45 & 0,6 \\
\hline
\end{tabular}

Tableau 6. Composition chimique des glaçures bleues

- Données PIXE exprimées en \% d'oxydes

\begin{tabular}{|l|l|l|l|l|l|l|l|l|l|l|l|l|l|l|l|l|l|}
\hline $\mathrm{N}^{\circ}$ d'inventaire & $\mathrm{Na}_{2} \mathrm{O}$ & $\mathrm{MgO}$ & $\mathrm{Al}_{2} \mathrm{O}_{3}$ & $\mathrm{SiO}_{2}$ & $\mathrm{~K}_{2} \mathrm{O}$ & $\mathrm{CaO}$ & $\mathrm{MnO}$ & $\mathrm{Fe}_{2} \mathrm{O}_{3}$ & $\mathrm{CoO}$ & $\mathrm{NiO}$ & $\mathrm{CuO}$ & $\mathrm{ZnO}_{2}$ & $\mathrm{As}_{2} \mathrm{O}_{5}$ & $\mathrm{SnO}_{2}$ & $\mathrm{Sb}_{2} \mathrm{O}_{5}$ & $\mathrm{PbO}$ & $\mathrm{B}_{2} \mathrm{O}_{3}$ \\
\hline MP 1876.304 bis & 0,68 & 0,59 & 2,07 & 38,00 & 2,14 & 3,00 & 1,07 & 1,17 & 0,41 & 0,23 & 0,28 & 0,09 & 0,06 & 0,05 & 0,06 & 49,43 & 0,1 \\
\hline 794.1.569 & 2,47 & 1,23 & 3,02 & 58,30 & 2,33 & 5,37 & 0,42 & 1,81 & 0,83 & 0,26 & 0,30 & 0,07 & 0,04 & 0,00 & 0,03 & 22,96 & 0,5 \\
\hline MP 1876.295 & 0,59 & 0,69 & 1,65 & 29,01 & 0,91 & 2,13 & 0,18 & 1,20 & 0,52 & 0,27 & 0,24 & 0,04 & 0,23 & 0,74 & 0,08 & 60,43 & 0,5 \\
\hline MP 273 & 0,79 & 0,50 & 0,70 & 29,74 & 3,10 & 3,17 & 0,16 & 1,05 & 0,62 & 0,47 & 0,18 & 0,04 & 1,01 & 0,01 & 0,20 & 57,43 & $<0,1$ \\
\hline MP 1876.290 & 1,09 & 0,59 & 1,05 & 40,11 & 1,90 & 3,50 & 0,21 & 1,69 & 1,28 & 0,74 & 0,28 & 0,09 & 1,77 & 0,00 & 0,07 & 44,89 & \\
\hline MR 3528 & 2,10 & 2,24 & 1,22 & 42,44 & 1,68 & 5,18 & 0,35 & 0,94 & 0,43 & 0,21 & 0,28 & 0,04 & 0,73 & 0,00 & 0,06 & 41,03 & 1,1 \\
\hline 794.1.570 & 1,40 & 0,13 & 0,81 & 34,83 & 2,29 & 0,77 & 0,05 & 0,43 & 0,43 & 0,14 & 0,65 & 0,19 & 0,66 & $\mathrm{ND}$ & 0,09 & 54,89 & 13 \\
\hline
\end{tabular}


œuvres se distinguent néanmoins. Pour le Neptune d'Amiens, la fritte au cobalt est enrichie uniquement au potassium. Sur l'aiguière de Rennes, les bleus sont caractérisés par des teneurs en bore supérieures à $10 \% \mathrm{~B}_{2} \mathrm{O}_{3}$ et le cobalt ne semble lié à aucun autre élément, ce qui laisse supposer qu'il aurait été introduit dans le mélange glaçurant non pas sous forme d'une fritte, mais sous forme d'oxydes.

Il est également important de considérer les éléments métalliques qui sont naturellement associés au cobalt dans les gisements, fer, nickel et arsenic entre autres. Ici, à l'exception de l'aiguière, les bleus des six autres pièces contiennent systématiquement du nickel. L'arsenic est également fréquemment détecté, sauf dans les bleus de la fontaine rustique et du plat de Rennes (794.1.569).

Dans le Corpus I, nous avions mis en évidence la même diversité des compositions de glaçures bleues. Dans la majorité des œuvres, la présence de quantités plus élevées de Na, $\mathrm{K}$, Ca ou Mg signe l'utilisation d'une fritte bleue. En revanche, les rapports alcalins/alcalino-terreux sont plus variables, probablement à cause de la diversité des supports de la matière colorante (smalt, verre bleu ancien recyclé, fritte de verre commerciale, minerai plus ou moins affiné, etc.). Mais nous n'y avons pas trouvé la composition potassique (smalt, verre potassique coloré en bleu par le saffre ?) comparable à celle du Neptune. La très grande majorité des pièces présente un cobalt lié à du nickel, la moitié contient en plus de l'arsenic.

Il est intéressant de constater qu'on trouve du cobalt " pur » dans de très rares cas, dans deux des plats représentant Henri IV et sa famille (MNC 6016 et ms 86-1-4), le plat signé de Pull et un essai de couleur bleu marine de la palette d'Avisseau, qui est le seul à contenir en plus de fortes teneurs en bore.

\section{Discussion}

Si l'on s'en tient aux sept œuvres de référence issues des saisies révolutionnaires et napoléoniennes (ensemble REF), six pièces apparaissent proches (la dernière, l'aiguière, très différente, fera l'objet d'un développement ultérieur). Toutes sont caractérisées par la présence de bore, dont la teneur, qui ne dépasse jamais $2 \%$, est le plus souvent de l'ordre de $0,5 \%$ $\mathrm{B}_{2} \mathrm{O}_{3}$; elles sont ainsi comparables à l'essentiel des 27 œuvres du Corpus I. Toutefois, des différences, souvent subtiles, soit dans les rapports entre les éléments majeurs (silice, plomb, alumine), soit dans les colorants (métaux liés au cuivre ou au cobalt), soit encore dans les frittes bleues incitent à analyser de façon plus approfondie les résultats.

Si l'on considère l'ensemble des données obtenues pour les pièces de référence, on remarque en premier lieu des variations dans les teneurs en Al. Elles concernent d'abord les glaçures colorées en jaune et en vert, souvent plus riches en aluminium que les autres glaçures d'un même plat. Pour les jaunes de trois des pièces (MP 1826.290, 794.1.569 et 1876.304 bis), les plus fortes teneurs en alumine liées à des teneurs en titane de $0,5 \%$ suggèrent que les céramistes ont intensifié cette couleur en ajoutant des argiles ferrifères. Pour deux autres œuvres en revanche (MP 273, MP 1876.295), ce serait le plomb qui jouerait ce rôle. Dans les glaçures vertes, les teneurs en alumine plus élevées auraient pour objectif non pas d'intervenir sur la couleur, mais plutôt sur la viscosité du mélange glaçurant : les potiers traditionnels ajoutent de l'argile (environ $10 \%$ ) pour obtenir à peu près $4 \%$ d'alumine dans la glaçure résultante afin d'éviter les coulures, notamment en présence de cuivre ${ }^{13}$.

Dans le cas de l'aluminium des glaçures incolores, une autre explication peut être proposée. Les récents travaux expérimentaux menés dans le cadre de la thèse de Gauthier Roisine $^{14}$, notamment par Emmie Beauvois ${ }^{15}$, montrent que l'aluminium peut diffuser depuis la pâte de façon non négligeable, enrichissant la glaçure en aluminium sur plusieurs dizaines de micromètres dans les proportions que nous avons mesurées sur les œuvres. La composition chimique résulterait donc non de l'ajout volontaire d'un ingrédient spécifique (argile), mais plutôt de processus de cuisson couplés à une faible épaisseur totale de glaçure. Cela semble être le cas quand les glaçures montrent un enrichissement notable en alumine, sans toutefois présenter de modifications des teneurs en titane, élément naturellement associé à l'aluminium dans les argiles. Nous poursuivons cette recherche, mais dans l'état actuel des connaissances, nous ne proposerons pas dans cet article d'explications définitives.

Quelles différences pouvons-nous établir dans les « recettes » des verts ou des bleus? Pour la couleur verte, trois types d'agent colorant apparaissent dans l'ensemble des pièces analysées. On sait que Bernard Palissy utilisait un cuivre assez pur. Cette recette ne se retrouve dans aucune des sept références qui, pour quatre d'entre elles (794.1.569, MP 1876.290, MP 1876.304 bis, MP 1876.295), mettent en œuvre un cuivre allié à de faibles quantités de zinc (rapport $\mathrm{Cu} / \mathrm{Zn}>10$ ) ; pour les trois autres (l'aiguière de Rennes, le Neptune d'Amiens, le plat dit « de la Tempérance»), le cuivre est associé à de plus fortes teneurs en zinc, comme ce que l'on trouve dans les ouvres plus récentes de Pull par exemple. Piccolpasso ${ }^{16}$ et d'autres céramistes du xvi ${ }^{\mathrm{e}}$ siècle, repris par Brongniart ${ }^{17}$, détaillent les opérations successives requises pour obtenir le cuivre nécessaire à la coloration d'une glaçure : récupérer du cuivre allié ou non, le meilleur étant le cuivre rouge (probable allusion au cuivre pur), le calciner après l'avoir mis en contact prolongé avec de l'urée ; la poudre qui en résulte est ajoutée au mélange glaçurant. Les variations cuivre/zinc, avec ou sans traces d'étain ou d'arsenic, résultent donc probablement du type de métal initialement utilisé. Il semblerait qu'il s'agisse plutôt de laiton quand le rapport $\mathrm{Cu} / \mathrm{Zn}$ est très bas. Mais ce rapport pourrait aussi être fonction du type de préparation du pigment (attaque chimique, différentes températures de calcination ou autre). Il est possible que les céramistes récupéraient du métal chez des dinandiers.

Pour les bleus, les données sont plus complexes à analyser. Si les observations restent les mêmes pour l'essentiel des 
œuvres, des différences assez subtiles ont été mises en évidence dans les frittes utilisées. Outre les deux pièces déjà isolées, l'aiguière de Rennes et le Neptune, la fontaine semble également se différencier par ses teneurs en potassium assez élevées. On ne peut pas, comme pour les Della Robbia, mettre en évidence de jalon chronologique en se basant sur la présence d'arsenic associé au cobalt ${ }^{18}$. Deux types de bleus, avec et sans arsenic, coexistent manifestement, même si l'association As/ Co/Ni semble prédominante. Les travaux plus systématiques en cours sur les glaçures de Palissy bleues et noires par N. Gandolfo et Y. Coquinot devraient permettre d'en savoir plus sur cette question ${ }^{19}$. Il faut aussi garder à l'esprit que les frittes bleues peuvent avoir des origines diverses.

Ainsi, à l'issue de cette phase des recherches, une lecture plus affinée des résultats obtenus sur l'ensemble REF peut être proposée avec un groupe d'œuvres largement majoritaire aux caractéristiques maintenant bien établies. Parmi elles, trois pièces (plat MP 1876.295, bassin d'aiguière MR 3528 et fontaine MP 1876.304 bis) se distinguent par des variations dans le choix des pigments ou dans la composition des mélanges. Ces différences étant souvent assez subtiles, on choisit pour le moment de les conserver dans ce groupe.

Le Neptune (MP 273) doit lui aussi être isolé avec ses recettes spécifiques, plus riches en plomb, moins alumineuses, des concentrations en oxydes colorants systématiquement plus élevées, et, pour le bleu, l'ajout d'une fritte potassique (ou du smalt ?) qui n'est pas reconnue dans d'autres pièces.

Enfin reste l'énigmatique aiguière (794.1.570), seule pièce des saisies révolutionnaires à présenter dans les glaçures bleues et blanches des teneurs en bore supérieures à $5 \%$ $\mathrm{B}_{2} \mathrm{O}_{3}$. Jusqu'à présent, de telles concentrations n'avaient été détectées que dans deux plats à rustiques figulines proposés comme tardifs (xIx ${ }^{\mathrm{e}}$ siècle?) ${ }^{20}$ : le MNC 3145 (voir fig. 5 de l'article de P. Lehuédé $e t$ al. dans ce volume) et peut-être le MR 2293, ainsi que dans un test de glaçure bleue sur une des palettes du céramiste Avisseau ${ }^{21}$. L'aiguière présente aussi d'autres particularités : un vert à forte teneur de zinc, l'absence d'indices d'utilisation d'une fritte pour les bleus et la présence d'un cobalt «pur ». Ce sont autant de paramètres qui signeraient une création tardive, si l'on n'avait la preuve de son existence avant la fin du XVIII ${ }^{\mathrm{e}}$ siècle. Elle est isolée dans notre corpus et provient donc indéniablement d'un atelier très différent des autres. Si l'on maintient une datation avant 1789, elle pourrait alors provenir d'un atelier, d'une manufacture, très au fait des dernières innovations de la chimie : le bore n'entre en effet réellement dans la composition des glaçures qu'à partir du XvirI ${ }^{\mathrm{e}}$ siècle ${ }^{22}$ et le cobalt a été isolé en 1735. La question reste entière pour le moment, mais ces données nous forcent à interpréter plus prudemment encore nos résultats, notamment en termes de chronologie.

La confrontation de ces données avec celles obtenues sur le Corpus I conforte l'idée d'un ensemble cohérent. En effet, la majorité des 27 pièces du Corpus I présentent des teneurs en plomb comprises entre 45 et $60 \% \mathrm{PbO}$, entre 30 et $45 \%$
$\mathrm{SiO}_{2}$, l'alumine y est souvent inférieure à $2 \%$; pour l'essentiel des œuvres, les taux d' $\mathrm{Al}_{2} \mathrm{O}_{3}$ augmentent dans les glaçures jaunes et vertes comme nous l'avons constaté, les plus faibles teneurs sont souvent enregistrées pour les glaçures bleues. Très souvent, le cuivre est associé au zinc dans des rapports compris entre 5 et 15 , il existe toutefois quelques pièces avec un vert très riche en zinc. Toutes ces caractéristiques se retrouvent dans le groupe majoritaire de l'ensemble REF. Il est intéressant de souligner que l'on peut y intégrer les œuvres attribuées au Maître du griffon et les céramiques au pied ocre du Corpus $\mathrm{I}^{23}$. Les compositions légèrement différentes des pigments verts et bleus du bassin d'aiguière MR 3528 et de la fontaine, semblent se retrouver dans deux ouvres du musée de Cleveland : une Sibylle de Cumes (inv. 1986-58) et un couronnement de Louis XIII (inv. 1986-51). Il est possible que ces quatre objets soient issus d'un atelier particulier ou qu'ils signent une chronologie spécifique. Mais ces différences de « recettes» de pigments ne pourraient-elles pas plutôt résulter d'approvisionnements variables selon les opportunités ? Les métaux cuivreux peuvent être recyclés, peu importe leur origine et leur qualité, un laiton, un bronze ou un cuivre, une fois calciné, donneront des verts satisfaisants ; les verres bleus peuvent être récupérés, une fois rebroyés et refondus, ils produiront un bleu acceptable pour autant qu'ils ne contiennent pas trop de manganèse ou de fer qui en altérerait la nuance finale.

Le Neptune (MP 273), lui, pourrait être rapproché de deux pièces, MNC 6016 et M 84.1.4, dont les glaçures contiennent peu d'alumine et dont les concentrations en oxydes métalliques sont particulièrement élevées.

Ainsi se dessine pour ces œuvres moulées une zone de production unique, probablement proche de Paris d'après les recherches de Jessica Denis-Dupuis (voir son article dans ce volume). Les petits groupes d'objets qui se différencient signeraient soit des ateliers différents, soit des périodes de production différentes. L'étude des pâtes argileuses, en cours, devrait aider à trancher entre ces hypothèses.

\section{Comparaison avec les statuettes}

Ces statuettes en ronde-bosse ont été souvent rattachées aux ateliers dits « d'Avon ${ }^{24}$ ", tout comme certaines œuvres examinées ci-dessus. Leur étude est donc intéressante pour amorcer une analyse comparative des matériaux et tenter de préciser leur origine. Nous n'en avons ici sélectionné que cinq (tableau $1 \mathrm{~b}$ ). La plupart présentent des caractéristiques très proches des céramiques décrites ci-dessus : une base de glaçure transparente à trois éléments principaux $\mathrm{PbO}$ (entre 50 et $60 \%), \mathrm{SiO}_{2}(30 \%), \mathrm{Al}_{2} \mathrm{O}_{3}(2$ à $5 \%$ ), l'absence d'opacifiants, des pigments traditionnels. C'est encore le bore qui va nous conduire à faire des distinctions au sein de ces objets, de même que les proportions relatives des trois constituants principaux. 


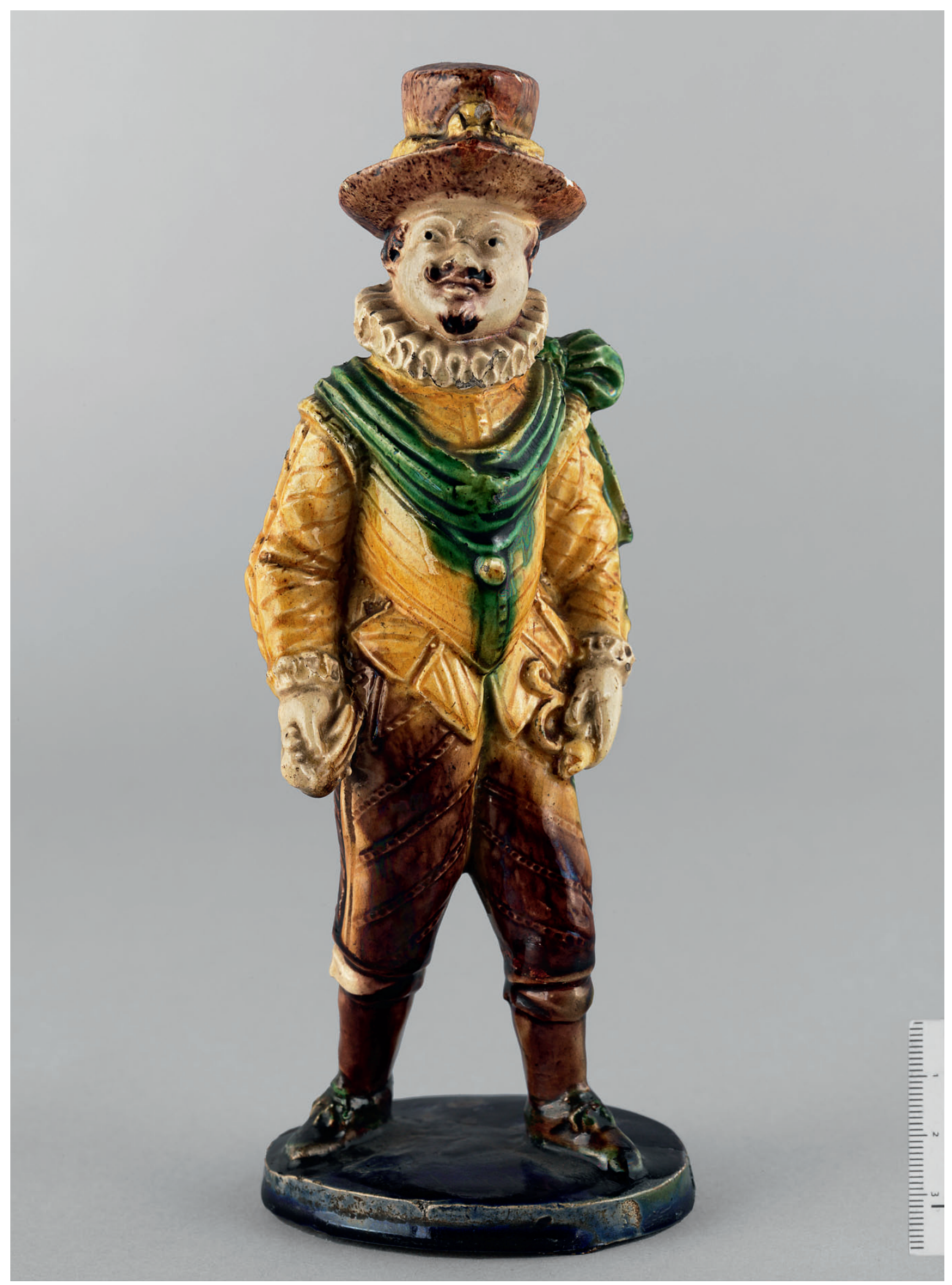

Fig. 5. Paris (?), première moitié du XVII ${ }^{\mathrm{e}}$ siècle, Statuette, Le Capitan, terre cuite à glaçure plombifère (H. 20,5 cm, L. $\left.8 \mathrm{~cm}, 1.8 \mathrm{~cm}\right)$, Paris, musée du Louvre, département des Objets d'art, MR 2370. ( C2RMF/A. Maigret. 


\section{Le Chien (OA 12071), le Personnage barbu assis en tailleur (OA 12072) et le Capitan (MR 2370) (fig. 3, 1 et 5)}

Ces trois pièces ont des compositions proches : $\mathrm{PbO}$ autour de 55-60\%, environ $30 \%$ de silice et 3 à $4 \% \mathrm{Al}_{2} \mathrm{O}_{3}$. Le bleu, quand il y en a, est obtenu à partir d'une fritte riche en calcium et en potassium, souvent peu sodique et dans laquelle le magnésium est peu abondant. Le cobalt y est associé au nickel et à l'arsenic. Le jaune est obtenu par du fer ; dans le vert, cuivre et zinc coexistent dans des proportions très variables d'une pièce à l'autre et on note parfois des traces d'arsenic. Les teneurs en bore sont inférieures à $1 \%$. Ces pièces s'intègrent dans le groupe majoritaire des saisies révolutionnaires décrit précédemment (cf. supra). Il est important de signaler que deux de ces statuettes proviennent de contextes archéologiques précis (maison du sabot) ${ }^{25}$.

\section{La Vache (MR 2368) (fig. 4)}

Ce sont des teneurs en bore très élevées (entre 3 et $6 \%$ $\mathrm{B}_{2} \mathrm{O}_{3}$ ) selon les couleurs qui caractérisent cette pièce. Si le rapport $\mathrm{PbO} / \mathrm{SiO}_{2}$ est assez proche de celui du groupe principal précédent, les teneurs en alumine, particulièrement basses, le distinguent. Les teintes sont très spécifiques, gris ou brun, et on ne peut que difficilement les comparer aux couleurs plus franches des sujets précédents. Il s'agit la plupart du temps de mélange dans des proportions variables des oxydes métalliques suivants : cuivre (sans association avec le zinc), manganèse ou traces de cobalt.

Pour le moment, cet objet (fig. 4) est isolé dans le lot des statuettes.

\section{Le Joueur de cornemuse (E.Cl. 13199)}

C'est un cas intéressant parmi l'ensemble des statuettes : la recette générale des glaçures est différente, moins plombifère et plus siliceuse, assez peu alumineuse (entre 1 et $2 \%$ $\left.\mathrm{Al}_{2} \mathrm{O}_{3}\right)$. Il n'y a pas de bore ou du moins dans des teneurs difficilement détectables par PIGE. Les teneurs en oxydes colorants sont plus élevées, créant des teintes très vives : plus de $7 \%$ pour le fer dans le jaune, près de $7 \%$ de cuivre associé à $2 \%$ de zinc dans le vert par ailleurs plus alumineux et, pour le bleu, une fritte de cobalt siliceuse, potassique et calcique ; le cobalt y est uniquement associé au nickel sans que l'on détecte d'arsenic.

Cette pièce (voir fig. 1 de l'article de P. Lehuédé et al. dans ce volume) est également isolée dans le lot des statuettes.

\section{Conclusion}

Dans la partie consacrée aux productions postérieures à Palissy publiée ici, seules les compositions chimiques élémentaires des glaçures ont été étudiées. L'ensemble des données montre une « signature » assez homogène avec la présence de traces de bore (entre 0 , 2 et $2 \% \mathrm{~B}_{2} \mathrm{O}_{3}$ ) probablement dues à des impuretés dans l'une des matières premières ${ }^{26}$. Les variations de teneurs en $\mathrm{SiO}_{2}, \mathrm{Al}_{2} \mathrm{O}_{3}$ et $\mathrm{PbO}$ (oxydes majoritaires) ou dans les caractéristiques des pigments seraient alors à rattacher à des ateliers spécifiques ou à des chronologies fines. On aurait alors pour ces œuvres, y compris pour une partie des petites statuettes, une zone de production unique, peut-être en région parisienne, comme le déduit Jessica Denis-Dupuis de ses études archivistiques. L'analyse des pâtes argileuses encore en cours devrait confirmer ou infirmer cette hypothèse.

Quelques pièces (le Neptune par exemple ou plus encore l'aiguière) montrent que d'autres ateliers, parfois très différents, coexistent. Ils pourraient avoir une production plus restreinte.

L'analyse de cinq statuettes ne permet qu'une toute première approche de l'étude des matériaux de ce type de productions et de leur origine. Dans l'état actuel des recherches, seules les deux pièces découvertes en fouilles et le Capitan se rattacheraient au groupe principal des saisies révolutionnaires. Mais la diversité des compositions enregistrées sur un si faible corpus augure bien de la complexité de la question des ateliers et plus encore de leur chronologie. Néanmoins, au fur et à mesure des analyses, se dessine, pour l'instant encore à très grands traits, le paysage de la céramique moulée post-palisséenne.

Une stratégie analytique plus complète s'impose maintenant. Elle doit comporter avant tout une analyse systématique des pâtes et un rapprochement avec des productions géographiquement bien ciblées. Il faut d'autre part composer de nouveaux corpus d'étude en privilégiant, au début du moins, des typologies homogènes. 
Notes

1. Voir l'article de F. Barbe et al. dans ce volume, Bouquillon et al., 2017 et 2018, Dupuis, 2011.

2. Tressaillage : réseau de fines fissures se formant dans la glaçure quand

la contraction de la couche vitrifiée est plus grande que celle du support

3. Bouquillon et al., 2018.

4. Voir l'article de J. Denis-Dupuis dans ce volume.

5. Voir l'article de F. Barbe et al. dans ce volume.

6. Bouquillon et al., 2017 et 2018.

7. Ce corpus rassemble des pièces du musée du Louvre, du musée national de la Renaissance à Écouen, du musée d'Art et d'Histoire de Lisieux et du musée des Beaux-Arts de Cleveland. Toutes correspondent à des œuvres moulées dont la glaçure et parfois la pâte ont été étudiées selon les mêmes protocoles que ceux mis en œuvre ici. L'ensemble des résultats est présenté dans deux articles de Bouquillon et al., 2017 et 2018.

8. Le protocole est le suivant : faisceau de proton de $3 \mathrm{MeV}$, flux d'hélium, quatre détecteurs Si-Li utilisés simultanément, l'un dédié à la détection des éléments légers (du sodium au cuivre) et trois autres équipés d'un filtre de 50 ou de $100 \mu \mathrm{m}$ d'Al, pour les éléments plus lourds (fer à uranium). Les éléments chimiques les plus légers, notamment le bore, sont mesurés par PIGE à l'aide d'un détecteur germanium. Chaque mesure dure environ trois minutes selon la dose choisie. Les résultats obtenus sont validés par l'analyse simultanée de standards géochimiques : NIST $1827 \mathrm{~b}$, NIST 93A, BGIRA 3 et 4, BRILL C, différents verres au plomb et DrN et parfois BEN (diorite et basalte du CRPG de Nancy). Pour mesurer les concentrations des éléments présents dans les matériaux étudiés, nous avons utilisé le logiciel GUPIX et l'application TRAUPIX (Calligaro, 2000). Les erreurs relatives sur les concentrations sont estimées à environ $5 \%$ (Radepont, 2017). Les teneurs en bore sont déduites de comparaisons avec des standards de verres SV 4001, SV 4002 et SV 4003 contenant respectivement 1,09, 0,84 et $0,02 \%$ B2O3. Plus d'informations sont données dans l'article de Castaing, Menu, 2006.

9. Bouquillon et al., 2018.

10. Bouquillon et al., 2017.

11. Voir l'article de P. Lehuédé et al. dans ce volume.

12. Bouquillon et al., 2018.

13. D’après Hamer et Hamer, 1997.

14. Bailly et al., 2017.

15. Beauvois, 2017.

16. Piccolpasso, 2007.

17. Brongniart, 1977.

18. Zucchiatti et al., 2006.

19. Travaux réalisés au C2RMF dans le cadre du projet « Palissy ».

20. Bouquillon et al., 2017.
21. Bouquillon, Pouthas, 2002.

22. Kingery, Vandiver, 1986.

23. Bouquillon et al., 2004, p. 101-103,

Bouquillon et al., 2017.

24. Voir l'article de J. Denis-Dupuis dans ce volume.

25. Le Chien et le Personnage barbu ont été trouvés lors des fouilles du Grand Louvre, voir Cat. Exp. Paris, 2001, p. 183.

26. Voir l'article de P. Lehuédé et al. dans ce volume.

\section{Bibliographie}

Bailly M., Bouquillon A., Durey V., Roisine G., 2017, «Les émaux de Bernard Palissy à l'épreuve de l'archéologie expérimentale ", Patrimoines : revue de l'Institut national du patrimoine, $\mathrm{n}^{\circ} 13$, p. 72-79.

Bouquillon A., Castaing J., Barbe F., Paine S. R., Christman B., Crépin-Leblond T., Heuer A. H., 2017, "Lead-glazed Rustiques Figulines (Rustic Ceramics) of Bernard Palissy (1510-1590) and his followers", Archaeometry, vol. 59, issue 1, p. 69-83.

Bouquillon A., Castaing J., Barbe F., CrépinLeblond T., Tilliard L., Paine S. R. Christman B., Heuer A. H., 2018, "French Decorative Ceramics MassProduced during and after the 17th Century: Chemical Analyses of the Glazes", Archaeometry, vol. 60, issue 5, p. 946-965, doi.org/10.1111/arcm.12349.

Bouquillon A., Castaing J., Calligaro T. et al., 2004, « Les suites de Palissy. Début d'enquête ", dans Cat. Exp. Lisieux, 2004, Bergeret J. (dir.), Les céramiques du Pré-d'Auge. 800 ans de production [Exp. Lisieux, musée d'Art et d'Histoire de Lisieux, 3 juillet 200430 septembre 2004], musée de Lisieux, p. 101-103.

Bouquillon A., Pouthas C., avec le concours de Musculus G. et Gratuze B., 2002 , «Les techniques des céramistes tourangeaux : analyses de laboratoire ", dans Cat. Exp. Tours, 2002, Le Leyzour P., Meslin-Perrier C., Oger D. (dir.), Un Bestiaire fantastique - Avisseau et la faïence de Tours - 1840-1910 [Exp. Tours, musée des Beaux-Arts, 19 octobre 2002-13 janvier 2003, Limoges, musée national Adrien-Dubouché, 4 février-12 mai 2003], RMN Éditions, Paris, p. 69-72 et annexes.

Brongniart A., 1977, Traité des arts céramiques ou Des poteries, fac-similé de l'édition de 1877, Dessain et Tolra, Paris.

Calligaro T., Dran J.-C., Poirot J.-P., Querré G., Salomon J., Zawaan J.-C., 2000, "PIXE/PIGE characterization of emerald using external microbeam", Nuclear Instruments and Methods In Physics Research B, 161-163, p. 769-774.

Castaing J., Menu M., 2006, "Analysis of art works and nuclear physics at the laboratory of Centre de recherche et de restauration des musées de France", Nuclear Physics News, 16, p. 4-10.
Cat. Exp. Paris, 2001, Bresc-Bautier G. (dir.), Le quartier du Louvre au XVII siècle, Archéologie du Grand Louvre [Exp. musée du Louvre, 2001], RMN Éditions, Paris.

Hamer F., Hamer J., 1997, The Potter's Dictionary of Materials and Techniques, $4^{\text {th }}$ ed., A \& C Black Publishers, London.

Kingery D., Vandiver P., 1986, Ceramic masterpieces: Art, structure and technology, Free Press, New York.

Piccolpasso C., 2007 (fac-similé), Les trois livres de l'art du potier qui traitent non seulement de la pratique mais aussi brièvement de tous ses secrets, matière toujours tenue cachée jusqu'à aujourd'hui, du chevalier Cipriano Piccolpasso, Casteldurante, présenté et traduit par J.-M. Lhôte, Vendin-le-Vieil, Éditions La Revue de la céramique et du verre.

Radepont M., Lemasson Q., Pichon L., Moignard B., Pacheco C., 2017, "Towards a sharpest interpretation of analytical results by assessing the uncertainty of PIXE/RBS data at the AGLAE facility”, Measurement 114, p. 501-507, dx.doi.org/ 10-1016/j. measurement.2016.07.005.

Roisine G., Capobianco N., Caurant D., Wallez G., Bouquillon A., Majérus O., Cormier L., Gilette S., Gerbier A., 2017, "The art of Bernard Palissy (15101590): influence of firing conditions on the microstructure of iron-coloured high-lead glazes", Applied Physics A, vol. 123, issue 8, 501, doi.org/10.1007/ s00339-017-1089-9.

Zucchiatti A., Bouquillon A., 2011, «Les glaçures : atout maître des Della Robbia ", dans Della Robbia - dieci anni di studi, SAGEP Editori, Gênes, p. 32-43.

Zucchiatti A., Bouquillon A., Katona I., D'Alessandro A., 2006, "The 'Della Robbia blue': a study case for the use of cobalt pigments in ceramics during the Italian Renaissance", Archaeometry, vol. 48, issue 1, p. 131-152, 6 ill. n. b., 7 tableaux, 11 graph.

\section{Documents inédits}

Beauvois E., 2017, Glaçures de la Renaissance : exemple des glaçures " Miel » de Bernard Palissy, mémoire de Master II, université de Bordeaux - Mention Archéologie, Sciences pour l'archéologie, 99 p.

Dupuis J., 2010-2011, La céramique d'Avon: limites et paradoxes d'une attribution héritée du XIX $X^{e}$ siècle, mémoire de Master I Recherche sous la direction de E. Lurin, université de Paris IV-Sorbonne, $231 \mathrm{p}$. 